FM 10:

Stellar explosions in an ever-changing environment 


\title{
Preface: Focus Meeting 10 - Stellar explosions in an ever-changing environment
}

\author{
Christina C. Thöne \& Lise Christensen \\ on behalf of the SOC of FM 10
}

\section{Background and aim of the meeting}

This focus meeting builds on a small conference "Galaxies meet GRBs at Cabo de Gata" held in September 2013 in Spain, which, for the first time, brought together people from the GRB and starburst communities and proved to be a great success. Focus Meeting 10 at the XXIX IAU GA was the continuation of this interdisciplinary collaboration, supported by Division J (Galaxies and Cosmology), Division D (High Energy Phenomena and Fundamental Physics) and Division G Working Group "Massive Stars".

The explosion of massive stars such as GRBs and SNe are among the most luminous events in the Universe. In the past decade the zoo of stellar explosions has expanded rapidly with a multitude of non-targeted transient surveys and will continue to do so. The continuos advance in computing facilities now makes it possible to simulate massive stars during their life and death in 3D and high resolution. The progenitors of some SN types have been determined thanks to large pre-imaging programs with the HST, though the largest and most intriguing SN Types still lack direct progenitor observations, some might even be inaccessible by tomorrow's telescopes.

The study of their immediate environment and hosts can to some extent replace direct progenitor observations. We are still in the beginning trying to determine what and if there are differences in the surrounding stellar populations of different explosions. Studying the hosts of objects such as GRBs has been a long and tedious process due to their distance and faintness and only now we start to have large enough samples for statistical studies. Looking at the global host properties might only make sense for the smallest of those hosts, e.g. those of GRBs and super-luminous SNe, hence it is more desirable to study their direct stellar environment. 3D spectroscopy of SN and GRB sites is another field still in its very infancy.

The direct impact of stellar explosions on their environment and galaxy evolution as a whole is still largely unknown, the same in the case of the impact on the evolution of element abundances. Due to the large timescales simulations are needed to give us some clues. Closing the circle between massive star formation, their explosive deaths and their influence back on their environment was the goal of Focus Meeting 10.

\section{Scientific Organizing Committee}

Focus Meeting 10 decidedly aimed at a predominantly young and gender-balanced organizing committee and invited speakers and we achieved an exact gender equality within both the SOC and invited speakers. The scientific organizing committee was composed of the following people:

Christina Thöne (Spain, chair), Lise Christensen (Denmark, co-chair), Susanna Vergani (France), Keiichi Maeda (Japan), Kris Belczynski (Poland), Nial Tanvir (UK), Lisa Kewley (Australia), Jason Prochaska (US), Francesca Matteucci (Italy), Avishay GalYam (Israel), Göran Östlin (Sweden), Emily Levesque (US), Polychronis Papaderos (Portugal). 


\section{Overview of the meeting}

In line with the SOC and invited speakers, the audience and contributed speakers were in majority young researchers and many engaged in lively discussions following each talk. We chose to allow as many people as possible to present their work and therefore opted for a large number of short talks which proved to be highly successful. We received over 50 requests for talks and could accommodate 33 in addition to 12 invited speakers.

The meeting consisted of the following sessions:

- The progenitors of stellar explosions (chairs: G. Meynet, J. Anderson and D. Szecsi)

- Starbursts across the Universe (chair: L. Kewley)

- The hosts of stellar explosions (chairs: F. Matteucci, A. de Ugarte Postigo)

- The hosts of stellar explosions - resolving the explosion site (chair: S. Vergani)

The progenitors of stellar explosions: Chris Fryer and Selma de Mink gave the introductory invited talks on theoretical models of single and binary progenitors of stellar explosions. While observations of stellar explosion remnants indicate that the models are not far from the truth, the situation regarding binaries and their influence on stellar evolution is still a largely unknown factor. Nancy Elias-Rosa and Giorgos Leloudas then showed us the current state of the SN progenitor observations and the properties of super-luminous supernovae (SLSNe) whose progenitor stars are still undetected. In a large number of contributed talks in this session we learned about how to determine SN (progenitor) properties from any wavelength range by studying the explosion itself or its remnant. Theoretical models on stellar evolution are critical to understand how stars explode and how does it relate to what we observe.

Starbursts across the Universe: Many hosts of stellar explosions are starburst galaxies and the short lifetime of massive stars usually imply that the starburst is still ongoing. Kelsey Johnson as substitute for Janice Lee gave an in-depth introduction to starbursts and star-formation processes on galactic scales at low redshift while her successor, Masami Ouchi, told us about star-formation rate density and Ly $\alpha$ halos at very high redshift by observing gravitationally lensed galaxies. Star-formation depends on the presence of molecular gas, but the first observations of GRB hosts by Michal Michalowski might indicate that stars also form out of HI gas. Unfortunately, this session attracted less talks than desired, possibly due to the parallel symposium on the high redshift Universe.

The hosts of stellar explosions: The session started of with a general introduction on absorption and emission properties of long-duration GRB hosts (those connected to the death of massive stars) by Paul Vreeswijk. Several contributed talks presented the large samples of GRB hosts, which have been put together just recently, such as BAT6, SHOALS or the X-shooter spectral sample. Chiaki Kobayashi presented her work on the modeling of abundances in star-forming galaxies across the Universe and the impact by stellar explosions. Two contributed talks followed up on this looking at the abundances in GRB hosts from both a theoretical and observational perspective. The session ended with an interesting talk by Lisa Kewley on emission line diagnostics, their changes with redshift (yes, they do change!) and where we have to be careful applying those diagnostics.

The hosts of stellar explosions - resolving the explosion site: Sebastian Sánchez, CALIFA PI, and Emily Levesque gave us an overview on resolved properties of star-forming galaxies in general and GRB/SN hosts in particular. The following contributed talks presented us the recent work in this relatively new field on the properties of SN Type II, Ic, broad-line Ic and GRB environments, mostly, but not only using 3D spectroscopy.

The talks of FM 10 will be permanently kept under the following link: www.iaa.es/iau2015_fm10/program.html 


\section{Poster contributions}

1. Kyle Augustson Super-equipartition Convective Dynamo Action in the Cores of B-Type Stars

2. Aldo Batta Accretion Disks and Sub-critical Angular Momentum in Collapsars

3. Tomasz Bulik Evolution of stars in the galactic center

4. Jon Hakkila The Complex Evolution of Gamma-Ray Burst Pulses

5. Nobuyuki Kawai $i W F-M A X I$ : Soft X-ray Transient Monitor on the ISS

6. Xiangcun Meng A common-envelope wind single degenerate model for SNe Ia

7. Bert Rust Calibrating the Decline Rate-Peak Luminosity Relation for Type Ia SNe

8. Kenji Tanabe Possible Geological Records of the Symbiotic Binary R Aquarii's Historical Outbursts

9. Naveen Yadav Dynamics of supernova driven superbubbles

10. Xin Zhou Star-formation activity around supernova remnants in our Galaxy -SNR G12\%.1+0.5 and SNR HB3

11. Takahiro Aoki Discovery Rate of Radio Transients with a Timescale of Minutes to Days; Need for the SKA-survey Antennas

12. David Corgan The Ghostly Novae of Spirits: Candidates, Confirmations, and Classifications

13. Nancy Elias-Rosa Catching Supernova Impostors

14. Ryan Hamilton Observations of the Type Ia Supernova SN2014J with FLITECAM/SOFIA

15. Christina Lacey Massive Star Formation in the Fireworks Galaxy: A High Resolution JVLA Survey of NGC 6946

16. Sangwook Park Mapping X-Ray Ejecta Distribution in the Galactic Core-Collapse SNR G292.0+1.8

17. Geraldine Peters The Abundances of the Fe Group Elements in Early B Stars in the Magellanic Clouds and Our Galaxy

18. Giuliano Pignata Supernova rates from the SUDARE survey

19. Katalin Takats SN 2009mw: a member of the tiny group of 1987A-like supernovae

20. Hideyuki Umeda Evolution and explosion of very massive metal-poor stars

21. N. Vasquez Alternative temporal classification of long Gamma Ray Bursts

22. B. Wang The progenitors of type IIn supernovae 